\section{Aterectomia Rotacional Multiarterial em Lesão de Tronco Esquerdo Seguida de Implante de Stents}

Bruno Macedo de Aguiar1, José Carlos Raimundo Brito Artigo original publicado em Rev. Bras. Cardiol. Invasiva. 2017; Isabela Pilar Moraes Alves de
Souza, Bruno Macedo Aguiar, Joberto Pinheiro Sena, Paulo José Bastos Barbosa, José Carlos

\section{INTRODUÇÃO}

A aterectomia rotacional (AR) foi desenvolvida com o objetivo de abordar placas ateroscleróticas calcificadas ainda na era da angioplastia transluminal coronária por cateter baláo, no final da decada de 1980 . Com o surgimento do stent, na década de 1990, a maioria das técnicas ateroablativas, incluindo a $A R$, caiu em desuso ou foi abandonada'. Na era dos stents farmacológicos (SF), a AR ressurgiu com o propósito de minimizar a expansão inadequada e/ou a má aposição do dispositivo, fatores estes relacionados com desfechos clínicos adversos, como a trombose e a reestenose do stent. No entanto, atualmente seu uso é infrequente, correspondendo a menos de $5 \%$ das intervenções coronárias percutâneas $(\mathrm{ICP})^{2,3}$.

\section{RELATO DE CASO}

Trata-se de uma paciente do sexo feminino, de 82 anos, com historia de angina há 10 anos e piora nos últimos 5 meses. Apesar do tratamento antianginoso otimizado, foi internada com infarto agudo do miocárdio sem supradesnivelamento do ST. Era portadora de hipertensão arterial sistêmica, dislipidemia, artrite reumatoide, insuficiência renal crônica não dialítica, anemia crônica sem causa identificada e episódios de fibrilação atrial paroxística.

O eletrocardiograma mostrava bradicardia sinusal e sobrecarga de átrio esquerdo. A radiografia do tórax evidenciava aumento de área cardiaca e leve congestão pulmonar. O ecocardiograma transtorácico indicava alteração segmentar da contratlidade do ventrículo esquerdo (VE) e FE de 39\%. Observou-se também estenose valvar aortica de grau moderado (gradiente médio de $31 \mathrm{mmHg}$ ). A cintilografia miocárdica mostrou isquemia moderada em região medial e apical da parede anterosseptal, além de isquemia grave em região medial e apical da parede infero-lateral do VE.
A coronariografia evidenciou presença de graves lesões obstrutivas, intensamente calcificadas no segsegmentos proximais e médios da descendente anterior (DA) e circunflexa (CX), em toda extensão da coronária direita (CD) e segmento proximal de ramo descendente posterior (DP) (fig. 1 e 2). O SYNTAX escore de 56. O escore da Society of Thoracic Surgeons (STS) calculado foi de $15,5 \%$ para mortalidade. O caso foi discutido pelo Heart Team, paciente e familiares e, em razão do ato risco cirúrgico, foi decidido por ICP estagiada. As ICPs ocorreram no intervalo de 15 meses, sendo a coronária esquerda tratada inicialmente (fig. 1). Foi realizada AR para possibilitar o implante de cinco SFs (total de $91 \mathrm{~mm}$ ) com sucesso. Feita a técnica de kissing stent na bifurcação do TCE e utilizado ultrassom intracoronário (USIC) para guiar o procedimento. $\mathrm{Na}$ evolução hospitalar, houve elevação assintomática de marcadores de necrose miocárdica e infecção respiratória que foi tratada, motivos que determinaram um tempo de internamento de 25 dias. Após 6 meses, apresentou retorno da angina, à despeito de otimização medicamentosa.

No segundo procedimento (fig. 2), após comprovação angiográfica do sucesso do procedimento anterio a $C D$ foi abordada por AR seguida de implante quatro da por USIC, que confirmou o sucesso angiográfico a boa expansão das hastes dos stents em toda sua extensão. Após o procedimento, ocorreu nova elevação de marcadores de necrose miocardica, sem alteração eletrocardiográfica, permanecendo assintomática do ponto de vista cardiovascular. Recebeu alta apos 3 dias e, em ecocardiograma transtoracico realizado 3 meses após esse procedimento, observou-se melho significativa da contratilidade segmentar e global do VE, com FE de $64 \%$. Atualmente, encontra-se assintomática e sem novos eventos cardiovasculares. mento distal do tronco da coronaria esquerda (TCE), SFs (total de $108 \mathrm{~mm}$ ). A intervenção também foi guia-
DISCUSSÃo

A calcificação coronária acentuada representa, ainda nos dias atuais, um importante desafio e limitação para ICP, dificultando a navegabilidade dos dispositivos para a abordagem das lesões e a expansão adequada dos stents. A má expansão dos stents pode estar associada à reestenose e nova revascularização da lesãoalvo, à trombose de stent e sua tradução clínica como infarto agudo do miocárdio e morte de etiologia cardía$\mathrm{ca}^{4}$. Alem disso, lesoes muito calcificadas podem representar uma ameaça especial para os SFs, podendo danificar o revestimento de polímero ${ }^{5}$, com a consequente difusão do antiproliferativo na parede do vaso, levando à redução da eficácia desses dispositivos.

Apesar da segurança e eficácia comprovada em estudos observacionais $s^{6-9}$ e do racional relacionado à modificação de placas muito calcificadas na ICP com implante de stents farmacológicos, a AR, quando utilizada de forma rotineira nesse cenário, não conferiu além de um alto índice de sucesso imediato do procedimento, benefícios clínicos adicionais consistentes em longo prazo, tais como redução da reestenose e/ ou eventos cardiacos adversos maiores. Isso foi demonstrado no estudo ROTAXUS ${ }^{10}$, o primeiro estudo randomizado que testou diretamente o impacto da AR associada ao implante de SF, nos desfechos de longo prazo. Nesse estudo, a utilização da AR de rotina não foi superior ao implante do SF sem previa AR, na redução do desfecho primário da perda tardia do lúmen intra-stent aos 9 meses de acompanhamento. Importante ressaltar algumas limitaçōes desse estudo como extensão media das lesões tratadas de apenas $20 \mathrm{~mm}$, ausência de calcificação importante em $40 \%$ dos pacientes que fizeram AR, grande taxa de perda de seguimento angiográfico $(20 \%)$ e falta de poder estatístico para comparar desfechos clínicos.

No caso clínico em questão, as lesões, além de severamente calcificadas, eram muito extensas, com perfil anatômico de maior complexidade comparado às do estudo ROTAXUS, sendo assim, a AR teve papel imprescindivel no sucesso do procedimento e na boa evoluçáo clinica da paciente. Vale ressaltar que, por ser uma técnica trabalhosa e que demanda alto nível de proficiência dos operadores, a AR deve fica reservada para grandes centros de excelencia na realização de ICP, que possuam dispositivos de imagem intravascular (USIC ou tomografia de coerência óptica), suporte circulatorio mecânico, cirurgia cardiaca de urgência e que, sobretudo, tenham grande experiência e capacitação para lidar com pacientes coronariopatas complexos e de alto risco.
Portanto, apesar de a AR não dispor de evidência científica robusta quanto aos seus benefícios na atua era dos SF, sua indicação ainda se faz presente numa minoria de casos selecionados, em pacientes portadores de doença arterial coronariana crônica, nos quais, despeito da presença de calcificação de grau moderado a importante das artérias e lesões obstrutivas, a ICP

FIGURAS

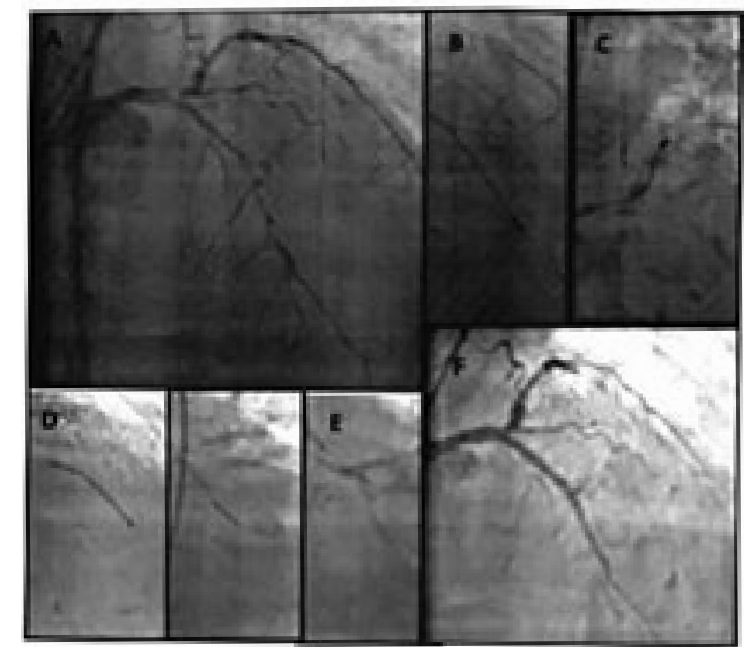

Figura 1 - A: graves lesões no TCE, DA e CX. B: aterectomia rotacional na DA. C: aterectomia rotacional na CX. D: implante de 2 stents na DA. E: implante de stents no TCE e CX. F: resultado angiográfico final.

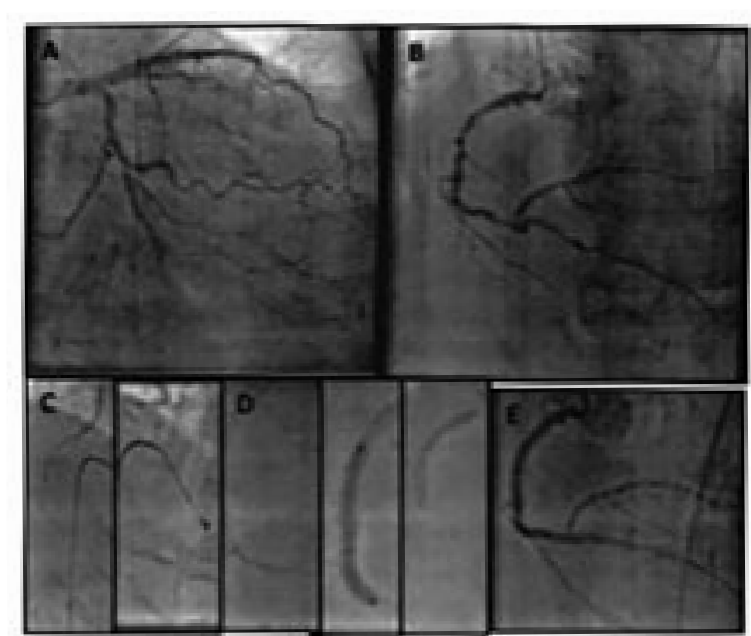

Figura 2 - A: sucesso angiográfico do procedimento anterior. B: graves lesões em toda extensão da CD. C: (D) CD. E: resultado angiográfico final. 


\section{REFERÊNCIAS}

1. Chen CC, Hsieh I-C. Application of rotational atherectomy in the drug-eluting stent era. J Geriatr Cardiol. 2013;10(3):213-6.

2. Baptista J. [Rotational atherectomy in the drug-eluting stent era: The revival of a forgotten technique?] Rev Port Cardiol. 2012;31(1):7-9. Portuguese.

3. Mota P, Santos R, Pereira H, et al. Facts on rotational atherectomy for coronary artery disease: multicentric registry (abstr). Paper presented at: EuroPCR; May 21, 2013; Paris, France.

4. Moses JW, Carlier S, Moussa I. Lesion preparation prior to stenting. Rev Cardiovasc Med. 2004;5 Suppl 2:S16-21.

5. Kuriyama N, Kobayashi Y, Yamaguchi M, Shibata $Y$. Usefulness of rotational atherectomy in preventing polymer damage of everolimuseluting stent in calcified coronary artery. J Am Coll Cardiol Intv. 2011;4(5):588-9.

6. Benezet J, Díaz de la Llera LS, Cubero JM, Villa M, Fernández-Quero M, Sánchez- González A. Drug-eluting stents following rotational atherectomy for heavily calcified coronary lesions: long-term clinical outcomes. J Invasive Cardiol. 2011;23(1):28-32.

7. Khattab AA, Otto A, Hochadel M, Toelg R, Geist V, Richardt G. Drug-eluting stents versus bare metal stents following rotational atherectomy for heavily calcified coronary lesions: late angiographic and clinical follow-up results. J Interv Cardiol. 2007;20(2):100-6.

8. García de Lara J, Pinar E, Gimeno JR, Hurtado JA, Lacunza J, Valdesuso R, et al. Percutaneous coronary intervention in heavily calcified lesions using rotational atherectomy and paclitaxel-eluting stents: outcomes at one year. Rev Esp Cardiol. 2010;63(1):107-10.

9. Dardas P, Mezilis N, Ninios V, Tsikaderis D, Theofilogiannakos EK, Lampropoulos S. The use of rotational atherectomy and drug-eluting stents in the treatment of heavily calcified coronary lesions. Hellenic $\mathrm{J}$ Cardiol. 2011;52(2):399-406.

10. Abdel-Wahab M, Richardt G, Büttner HJ, Toelg R, Geist V, Meinertz T, et al. High- speed rotational atherectomy before paclitaxel-eluting stent implantation in complex calcified coronary lesions: the randomized ROTAXUS (Rotational Atherectomy Prior to Taxus Stent Treatment for Complex Native Coronary Artery Disease) trial. JACC Cardiovasc Interv. 2013;6(1):10-9.

1- Serviço de Cardiologia Intervencionista do Hospital Santa Izabel

Endereço para correspondência:

bmaguiar@gmail.com 